\title{
The expression and significance of NET-1 and Contactin in hepatocellular carcinoma
}

\author{
Yunyao Ye ${ }^{1,2}$, Li Chen ${ }^{1 *}$, Guilan Wang ${ }^{1}$, Jin Qin ${ }^{1}$, Jiaming Zhou ${ }^{1}$, Tiejun Li ${ }^{3}$, York Yuanyuan Zhu ${ }^{3}$ \\ ${ }^{1}$ Department of Pathological Anatomy, Nantong University, Nantong, Jiangsu 226001, China; \\ ${ }^{2}$ Department of Tumor, Taizhou People's Hospital, Taizhou, Jiangsu 225309, China; \\ ${ }^{3}$ Biomics Biotechnologies Co., Ltd, Nantong, Jiangsu 226016, China
}

\begin{abstract}
Hepatocellular carcinoma (HCC) is one of the most common cancers worldwide and a major cause of cancerrelated mortality. In this study, the significance of NET and Contactin on the pathogenesis and prognosis of HCC was investigated, and further to explore their functions in vitro and in vivo by down regulation with siRNA. The expression of NET-1 and Contactin in HCC and in adjacent non-tumor tissues (ANT) were evaluated by immunohistochemistry, and the correlations of the expression of NET-1 and Contactin with the clinicopathological characteristics and survival of HCC patients were also analyzed. After inhibited by single-target siRNA or dualtarget siRNA, the expressions of NET-1 and Contactin mRNA and protein in SMMC-7221 cells were determined by RT-PCR, Western blot and immunofluorescence stain. The cell proliferation and apoptosis were assessed by CCK-8 assays and flow cytometry (FCM). The ability of cell migration and invasion were evaluated by woundhealing migrating assay and transwell chamber assays, respectively. Subsequently, transmission system encapsulated cationic liposome was used to deliver dual-siRNA into HCC xenografts in mice. The expressions of NET-1, Cortactin, Ki67, Bax, Bcl2 and Survivin in xenograft tumor were detected by immunohistochemical staining, respectively. The positive rates of NET-1 and Cortactin in HCC tissues were significantly higher than those in ANT. In HCC, the expression of NET-1 was related to Edmondson's grade $(P<0.05)$, cirrhosis background $(P<0.001)$ and TNM stage $(P<0.05)$. The expression of Cortactin was related to tumor infiltration $(P<0.05)$, vascular invasion $(P<0.001)$ and TNM stage $(P<0.001)$. The expressions of NET -1 and Cortactin were positively correlated $(r=0.280$, $P=0.004)$. Significant differences in the 5-year survival rates were seen between the NET-1 negative group and the NET-1 positive group $(P<0.05)$, and between the Contactin negative group $(50 \%)$ and Contactin positive group (28.0\%, $P<0.01)$. The 5 -year overall survival rate (OS) in NET-1 and Contactin co-expression cases (27.78\%) were remarkably lower than that in both NET-1 and Contactin negative cases (54.54\%) and in NET-1 positive while Contactin negative cases $(47.06 \%, P<0.05)$. Univariate and multivariate Cox regression analysis revealed that NET-1 and Contactin over-expression were independent indicators for OS in HCC patients $(P<0.01)$. There were higher expressions of NET-1 and Contactin in SMMC-7721 cells than that in other HCC cells. Dual-siRNA was demonstrated to be more effective on inhibiting cancer cell proliferation, migration and inducing apoptosis than individual siRNAs used alone in vitro and in vivo $(P<0.05)$. The results suggest that dual-siRNA may be a great potential in siRNA-based therapeutic applications.
\end{abstract}

Keywords: NET-1, Contactin, hepatocellular carcinoma, siRNA

*Correspondence to: Li Chen, Department of Pathological Anatomy, Nantong University, Nantong, Jiangsu 226001, China. TEL: +86-51385051729, FAX: +86-513-85051729, E-mail:bl1@ @tu.edu.cn. The authors declared that they do not have anything to disclose regarding funding or conflict of interest with respect to this manuscript. 


\section{INTRODUCTION}

Hepatocellular carcinoma (HCC) is the seventh most common cancer and the third leading cause of cancer related mortality in the world ${ }^{[1]}$. The incidence rate of HCC increased every year, especially in developing countries. It was estimated that 748,300 new cases and 695,900 deaths from liver cancer occurred in $2008{ }^{[2]}$. At present, the main therapeutic methods for HCC include surgical excision, liver transplantation and local ablation. HCC is known to be relatively resistant to systemic therapy, thus the prognosis for most patients, especially with multicentric recurrence and intrahepatic metastasis remains poor, and this is partially due to lack of effective method for early diagnosis and treatment ${ }^{[3-4]}$. Identifying the differentially expressed genes and investigating their characteristics will be beneficial to understand the molecular mechanism in the pathogenesis and progression of HCC and to produce more effective molecular targets for cancer treatment.

Both NET-1 and Contactin have been previously reported as cancer-associated genes, and therefore have the potential to be used as biomarkers for cancer detection and prognostic evaluation. NET-1 (TSPAN1 gene), a member of the transmembrane 4 superfamily or tetraspanin superfamily (TM4SF), has been identified as a signaling protein possessing important functions, such as cellular proliferation, migration and differentiation ${ }^{[5-6]}$. Cortactin (cortical actin-binding protein), encoded by EMSI (cytoskeleton recombinant related gene) was recently reported to be metastasisrelated ${ }^{[7-9]}$. Cortactin activates actin polymerization by binding to Arp2/3 complex; as a result, cell migration enhanced ${ }^{[10-11]}$. In addition, Contactin expression was highly correlated with portal vein embolus and extrahepatic metastasis ${ }^{[12-13]}$. Indeed, the overexpression of NET-1 and Contactin have been demonstrated in various types of human tumors, such as lung ${ }^{[14-15]}$, liver ${ }^{[12-13,16-18]}$, colorectal ${ }^{[19-20]}$, skin ${ }^{[21-22]}$ and cervical carcinomas ${ }^{[23]}$, and may assist tumor cells in continuing to grow.

Small-interfering RNA (siRNA, 21 nucleotides), normally generated from long double-stranded RNAs, are now frequently used to knockdown or silence specific genes to understand particular gene functions ${ }^{[24-25]}$. RNAi-based knockdown of specific oncogenes is believed to be a potential strategy for cancer treatment.

In this study, expressions of NET-1 and Contactin in $\mathrm{HCC}$ were detected by tissue microarray constructed by surgically-resected HCC tissues. And their correlations to clinicopathological features and prognosis were analyzed to elucidate the significance of NET-1 and Contactin in HCC. Furthermore, single target siRNAs (siRNA-NET-1 or siRNA-Contactin) and a dual target siRNA simultaneously knockdown both genes (dualsiRNA) were constructed to explore the functions of NET -1 and Contactin in HCC in vitro and in vivo.

\section{MATERIALS AND METHODS}

\section{Patients and follow-up}

The study population was comprised of 103 HCC patients who were diagnosed with HCC and had undergone curative resection in Nantong Tumor Hospital and the Affiliated Hospital of Nantong University between January 2003 and December 2004. The patients were selected according to the following criteria: (1) Primary HCC; (2) previously untreated, and with surgery the first treatment. Therefore, analysis of the data in this series would reflect the actual impact of the tumor biology on the clinical outcome.

Among the 103 patients, 85 were males and 18 were females, and the ratio of male to female was 4.8:1 $(82.5 \%$ and $17.5 \%)$. Age of the patients ranged from 21 to 79 years old with a median of 49 years old. All patients were diagnosed and histopathologically confirmed with HCC, and had complete clinical and pathological records, including patients' medical records, chest roentgenograms, and whole body computed tomography films, bone and brain scanning data. The records of surgery were reviewed, and the confirmed pathological diagnosis, tumor size, related hepatitis/ liver cirrhosis, metastasis, serum AFP values and other relevant data were analyzed retrospectively. Negative control was established from 57 matched adjacent nontumor ANT liver tissues which were derived from 103 cases of HCC. The study was approved by the Ethics Committee of Nantong Tumor Hospital and the Affiliated Hospital of Nantong University, and all the patients voluntarily joined this study and signed on informed consent.

All patients had regular follow-up, and the last follow-up time was January 2010. The follow-up period was defined as the duration from the date of operation to the date of either death or the last follow-up. In this study, the follow-up period ranged from 13 to 72 months with a median of 58.4 months. Deaths from other causes were treated as censored cases. Overall survival (OS) was evaluated by the duration from the dates of surgery to death. To determine the influence factors of survival after operation, conventional variables together with NET-1 and Contactin expression were determined in the 103 patients. 


\section{Preparation of tissue microarray}

Tissue microarrays were used in this study, which consisted of archived tissues retrieved from surgicalpathological files. In total, there were 57 matched ANT tissues and $103 \mathrm{HCC}$ tissues. Tissue microarrays were constructed as previously described (Qun E, Chen Li and Jing Guohua, patent number: ZL200810022170.4). Briefly, donor paraffin blocks were made to perform HE staining, which was made by pathologist under microscope in a double blind manner, The pathological diagnosis was recorded in detail for HCC Edmondson's grade and TNM stage according to the WHO description of HCC characteristics ${ }^{[26]}$. A sampling needle with a diameter of $1.6 \mathrm{~mm}$ was inserted into the paraffin block where the typical lesions and retrieved tissues were located. Two tissue cores were punched on each specimen. According to the amount of samples taken, tissue arrays were designed in the arrangement of $10 \times 12$ for the blank recipient block. The selected tissue cores were then inserted into a recipient block according to a pre-determined design for twice embedding to make it closely integrate with the sounding paraffin mass.

\section{Immunohistochemistry (IHC) staining}

Serial sections were cut at $4 \mu \mathrm{m}$ thickness from tissue microarrays, and dimethyl benzene was used to deparaffinage. Epitope retrieval was performed using microwave heat in citrated buffer $(10 \mathrm{mmol} / \mathrm{L}$ sodiumcitrate monohydrate, $\mathrm{pH} 6.0$ ). Endogenous peroxydase was blocked by $3 \% \mathrm{H}_{2} \mathrm{O}_{2}$, and then incubated with $10 \%$ calf serum. Polyclonal rabbit-anti-human NET-1 (Gene Biotech, CA, USA, 1:100 dilution) and mouse-anti-human Cortactin (Santa Cruz Biotech, USA 1:100 dilution) were incubated at $37^{\circ} \mathrm{C}$ for $30 \mathrm{~min}$ and at $4{ }^{\circ} \mathrm{C}$ overnight. The slides were incubated with biotin-conjugated antibody for $30 \mathrm{~min}$ at $37^{\circ} \mathrm{C}$ and streptomycin antibioticperoxidase solution for an additional $30 \mathrm{~min}$ at $37^{\circ} \mathrm{C}$, followed by staining with DAB and weakly counterstaining with Mayer's hematoxylin. SP immunohistochemistry kit and DAB coloration kit were purchased from Maixing Biotechnology Company (KIT-9710 and DAB-0031, Fuzhou, China). Phosphate-buffered saline (PBS) was used to replace the primary antibody as a negative control. Gastric carcinoma tissues with a known positive expression of NET-1 and Contactin were defined as a positive control independently. Pictures were taken for five visual fields in each section of the tumor.

Stained sections were evaluated using an immunoreactive score (IRS) in a blinded manner by two independent investigators without prior knowledge of the clinical information. Scores were assigned to the intensity and the percentage of positive staining of the cytoplasm/membranes in the whole cylinder. Briefly, the IRS assigns sub-scores for immunoreactive distribution $(0-4)$ and intensity $(0-3)$, then multiplies them to yield the IRS score. The percentage of positivity was scored as “0” (<5\%), “1” (5\%-25\%), “2” (>25\%$50 \%)$, “3" $(>50 \%-75 \%)$ or " 4 " (>75\%). The staining intensity was scored as " 0 ” (no staining), " 1 ” (weakly stained), “2” (moderately stained) or " 3 " (heavily stained). When the scores of duplicate tissue cores were different, the higher one was recorded. The final expression scores of NET-1 and Contactin were calculated by the value of the percentage of positivity multiply staining intensity, which ranged from 0 to 12 . Intratumoral antibody expression was defined as following: negative (-, score 0$)$, low expression (+, score $1-6)$ and high expression $(++$, score $>6)$.

\section{Cell culture}

Three human HCC cell lines (SMMC-7721, Huh7 and HepG2) were purchased from the Institute of Cell Biology (Shanghai, China) and maintained in Dulbecco's modified Eagle medium (DMEM, Invitrogen, USA) plus $10 \%$ fetal bovine serum (FBS, Invitrogen, USA), $100 \mathrm{U} / \mathrm{mL}$ penicillin and $100 \mu \mathrm{g} / \mathrm{mL}$ streptomycin (Invitrogen, USA) at $37^{\circ} \mathrm{C}$ in $5 \% \mathrm{CO}_{2}$ incubator. Cells with the highest expression of NET-1 or Contactin were selected for the following experiments.

\section{siRNAs and cell transfection}

The sequences of single-target siRNA against either $N E T-1$ or Contactin and dual-target siRNA (dualsiRNA) simultaneously against both genes were designed and synthesized by Biomics Biotechnologies Co. Ltd (China). A negative control siRNA (NC siRNA) was also used. These siRNAs were transfected into cells by Lipofectamine 2000 (Invitrogen, USA) respectively according to the manufacturer's instruction. The cells without siRNA transfection were used as mock control. All siRNAs sequences were shown in Table 1.

\section{Table 1 Sequences of single target-siRNA and dual-siRNA}

\begin{tabular}{ll}
\hline siRNA & \multicolumn{1}{c}{ Sequences $\left(5^{\prime} \rightarrow 3^{\prime}\right)$} \\
\hline NET-1 siRNA & Sense: CCACAAUGGCUGAGCACUUdTdT \\
& Antisense: AAGUGCUCAGCCAUUGUGGdTdT \\
Cortactin siRNA & Sense: GAACAAGACCGAATGGATAdTdT \\
& Antisense: TATCCATTCGGTCTTGTTCdTdT \\
dual-siRNA & Sense: CCACAAUGGCUGAGCACUUA GAACA \\
& AGACCGAAUGGAUAdTdT \\
& Antisense1: AAGUGCUCAGCCAUUGUGGdTdT \\
NC siRNA & Antisense2: UAUCCAUUCGGUCUUGUUCdTdT \\
& Sense: UUCUCCGAACGUGUCACGUdTdT \\
\hline & Antisense: ACGUGACACGUUCGGAGAAdTdT \\
\hline
\end{tabular}




\section{Real-time quantitative PCR (RT-qPCR)}

Total RNA was extracted from cells with Trizol reagent (Invitrogen, USA) for cDNA synthesis $48 \mathrm{~h}$ post-transfection. Reverse transcription was performed using AMV Reverse Transcription Reagent (Quantitech, USA). The sequences of the primers were listed in Table 2. PCR for DNA synthesis was performed as the following procedures: after a first denaturing step at $95{ }^{\circ} \mathrm{C}$ for $5 \mathrm{~min}, 40$ cycles consisting of denaturation at $95^{\circ} \mathrm{C}$ for $20 \mathrm{~s}$, annealing at $58^{\circ} \mathrm{C}$ for $30 \mathrm{~s}$, and extension at $72{ }^{\circ} \mathrm{C}$ for $30 \mathrm{~s}$. Real-time quantitative fluorescence PCR method was used to detect the mRNA expression levels. GADPH was used as an internal control.

Table 2 Sequences of the primers for RT-qPCR

\begin{tabular}{ll}
\hline Primers & \multicolumn{1}{c}{ Sequences $\left(5^{\prime} \rightarrow 3^{\prime}\right)$} \\
\hline NET-1 & Forward: GTGGCTTCACCAACTATACG \\
& Reverse: GACTGCATTAGTTCGGATGT \\
Cortactin & Forward: AGAGGCTGTCTATGAAAG \\
& Reverse: GATGTTGGTGATGATGTC \\
GADPH & Forward: GGTCTCCTCTGACTTCAACA \\
& Reverse: AGCCAAATTCGTTGTCATAC \\
\hline
\end{tabular}

\section{Western blot}

Cells were harvested $48 \mathrm{~h}$ after siRNAs transfection and lysed in ice-cold cell lysis in $2 \times$ sodium dodecyl sulfate (SDS) sample buffer $(0.5 \mathrm{~mol} / \mathrm{L}$ Tris-HCL pH6.8, $10 \%$ SDS, $5 \%$ glycerol) supplemented with $5 \%$ $\beta$-meraptoethanol. After centrifugation at $4{ }^{\circ} \mathrm{C}(13,000$ $\mathrm{rpm}, 15 \mathrm{~min}$ ), the proteins in lysates were analyzed using immunoblotting assay. The proteins were separated by $10 \%$ SDS-PAGE and transferred to PVDF membranes (Pharrnacai, USA). The membranes were incubated with primary antibodies of polyclonal rabbitanti-human NET-1 (1:1,000 dilutions) and mouseanti-human Contactin (1:2,000 dilutions) overnight respectively. After washing with $0.05 \%$ Tris-buffered saline/Tween-20 buffers (TBST, pH7.4), the membranes were incubated with secondary antibody $\operatorname{IgG}$ conjugated HRP (1:1,000 dilutions) for $2 \mathrm{~h}$ at room temperature. $\beta$-actin antibody was used to normalize protein expression. After being washed three times by TBST, the relative amount of proteins on the membranes was determined by densitometry using ScionImage 4.02 software.

\section{Immunofluorescence staining}

Cells were cultured on coverslips in 24-well plates, transfected with siRNAs, and incubated for $48 \mathrm{~h}$. Then the coverslips were washed twice with PBS, and fixed with $4 \%$ paraformaldehyde for $1 \mathrm{~h}$. The cells were permeabilized with $0.2 \%$ Triton $\mathrm{X}-100$ for 5 min, blocked with $1 \%$ bovine serum albumin (BSA) in PBS for $1 \mathrm{~h}$ at room temperature, and incubated overnight at $4{ }^{\circ} \mathrm{C}$ with polyclonal rabbit-anti-human NET-1 (1:100 dilutions) and mouse-anti-human Contactin (1:100 dilutions) respectively, then washed extensively, followed by labeling with FITC or TRITC labeled secondary antibody (1:100 dilutions) (Sigma-Aldrich, USA) for $2 \mathrm{~h}$ at $37^{\circ} \mathrm{C}$. The stained cells were observed using immunofluorescent microscopy.

\section{Cell proliferation assay}

The cells in logarithmic growth phase were cultured in a 96-well plate in triplicate wells, and allowed to grow up to $60 \%-80 \%$ confluence before transfection. After transfected with siRNAs, $100 \mu \mathrm{L}$ DMEM and $10 \mu$ L CCK-8 (Cell Counting Kit-8, Dojingo, Japan) were mixed and loaded into every well, and incubated for $4 \mathrm{~h}$. OD values of every wells were then obtained at $490 \mathrm{~nm}$ using a Microplate Reader (Bio-RAD, USA).

\section{Flow cytometric analysis}

Flow cytometry (FCM) was used to determine the cell apoptosis. Annexin-V/propidium iodide (PI) double staining assay was performed using the Annexin V-FITC Apoptosis Detection kit (KeyGEN Biotech, China). Briefly, transfected cells in the dishes were harvested with trypsin and washed twice with PBS. Cells $\left(1 \times 10^{6}\right)$ were resuspended in $500 \mu$ L binding buffer and stained with $5 \mu$ L FITC-labeled Annexin$\mathrm{V}$ according to the manufacturer's instructions. PI(5 $\mu \mathrm{L})$ was added and allowed to incubate with cells for $10 \mathrm{~min}$ at room temperature in dark. Five groups were subjected to FCM analysis using BD FACS Calibur (BD Bioscience, USA). The data were analyzed using CellQuest data acquisition and software (BD Bioscience, USA).

\section{Wound-healing migrating assay}

Transfected cells were grown in 96-well plates, and the monolayer of cells was scratched a lane across the bottom of each well. Cells were washed twice with serum-free DMEM medium gently to remove the floating cells. Then, $100 \mu \mathrm{L}$ of complete DMEM medium with $10 \%$ FBS was added to each well and incubated for $0,24,48$ and $72 \mathrm{~h}$. Relative migration distance was calculated using following formula: relative migration distance $=(A-B) / A \times 100$. $A$ represents the initial distance after scratching, and $B$ is the distance when the experiment finished.

\section{Transwell chamber migrating assay}

The cell invasion ability after transfection was compared among 5 groups using transwell chamber migration (Corning Bioscience, USA). Cells ( $1.5 \times 10^{5}$ cells in every chamber) were placed in the upper chamber of 
the membrane filters and allowed to penetrate filters. The lower chamber was filled with culture medium. The cells on the upper side of the membrane filters were removed with a cotton swab 48 hours later. And the cells that had migrated to the underside of the filters were fixed with $10 \%$ formaldehyde for $30 \mathrm{~s}$ and stained with crystal violet for 3-5 min immediately, followed by 3 washes with PBS. Cells were counted under 5 fields $(\times 200$ magnified $)$ in each filter.

\section{Xenograft tumor models}

Four-six-week old athymic ( $T$ cell deficiency) nude mice (BALB/c Nude) were provided by the Animal Experiment Center of Nantong University. The cells used to generate subcutaneous xenograft $\left(1 \times 10^{7}\right.$ cells in $0.2 \mathrm{~mL}$ basic culture medium) were injected s.c. into the nape of the mice. After 14 days, the mice with tumors growing on their subcutaneous flank were divided into 3 groups with 4 mice in each group, the dualsiRNA group was intratumour multipoint injected with the delivery system enhanced by cationic lipids packaging together with dual-siRNA, negative dual-siRNA (NC siRNA) obtained from the Nantong Bionics Biotechnology Corporation was used as negative control, and equal volume $(38 \mu \mathrm{L})$ of PBS was injected to the untreated control group, all mice were injected every four days and were euthanized 30 days after xenograft injection. Tumor volume was calculated by the following formula: $V=A B^{2} / 2\left(\mathrm{~mm}^{3}\right)(A$ and $B$ represent the maximum diameters, respectively).

\section{Histological observation}

The paraffin sections of tumor tissues were stained with H\&E and IHC, respectively. The primary antibodies included rabbit anti-human NET-1, mouse antihuman Cortactin, and antibodies for Ki67, Bax, Bcl2, Survivin (DAKO, USA, 1:100 dilutions, respectively). The process of IHC staining was as described above.

\section{Statistical analysis}

SPSS 17.0 software (SPSS Inc, USA) was used for all statistical analyses. Chi-square $\left(\chi^{2}\right)$ test and Spearman rank correlation test were used for comparison of the difference between HCC and ANT or the association of NET-1 and Contactin expression with clinicopathologic parameters of HCC. Curves for OS were drawn according to the Kaplan-Meier method and difference was analyzed by applying the log-rank test for univariate survival analysis. In accordance with results driver from Cox univariate regression analyses, significant factors were furthermore evaluated by multivariate regression analyses to determine independent prognostic factors. In vitro all groups and assays were performed repeatedly three times. All results were repressed as mean \pm SD and statistical differences were calculated using a standard oneway ANOVA and two-tailed unpaired Student's $t$ test. $P<0.05$ was considered as statistically significant.

\section{RESULTS}

\section{Expression of NET-1 and Contactin in HCC tissues and ANT tissues}

The IHC results of liver tissue arrays confirmed the expression of NET-1 and Contactin protein which was brownish yellow, and located in cytoplasm and/or cell membranes. In this study, the differences of NET1 and Contactin expression between HCC tissues and matched ANT were observed in 57 samples (Table 3). The positive rates of NET- 1 and Contactin in HCC tissues were $85.96 \%$ (49/57) and $73.68 \%$ (42/57), respectively, which were significantly higher than matched ANT $[59.6 \%(34 / 57)$ and $28.1 \%(16 / 57)]\left(\chi^{2}=8.332\right.$ and 29.991, $P<0.001)$. In HCC tissues, NET-1 expressed intensively in the marginal cells of cancer nests compared with the center of cancer nests. Cancer cells with diffusely infiltrated stroma and vessels showed strongly positive expressions of Contactin (Fig. 1,2).

Table 3 NET-1 and Contactin expression in HCC and matched ANT (57 cases)

\begin{tabular}{cccccccc}
\hline \multirow{2}{*}{ Sample } & \multicolumn{3}{c}{ NET-1 } & & & \multicolumn{3}{c}{ Cortactin } \\
\cline { 2 - 4 } \cline { 6 - 8 } & - & + & ++ & & - & + & ++ \\
\hline HCC & 8 & 27 & 22 & 12 & 21 & 24 \\
ANT & 23 & 29 & 5 & 41 & 9 & 7 \\
\hline
\end{tabular}

\section{Association of NET-1 and Contactin expres- sion with clinicopathologic parameters of HCC}

The correlations of NET-1 and Contactin expression with the clinicopathologic characteristics of HCC were showed in Table 4. The intratumoral NET-1 expression was significantly correlated with HCC Edmondson's grade $\left(\chi^{2}=11.424, P=0.003\right)$, TNM stage $\left(\chi^{2}=16.434, P=0.012\right)$, HCC with cirrhosis background $\left(\chi^{2}=38.888, P<0.001\right)$ and tumor sizes $\left(\chi^{2}=19.370\right.$, $P=0.001)$. The NET -1 positive rate in Edmondson III - IV grade $94 . .44 \%(51 / 54)$ was higher than that in I II grade $77.55 \%(38 / 49)$, in TNM III - IV stage $94.23 \%$ (49/52) was higher than that in I - II stage $78.43 \%$ (40/51), in HCC with cirrhosis background $96.38 \%$ (80/83) was higher than that without cirrhosis $45.0 \%$ $(9 / 20)$, in tumor size $2-10 \mathrm{~cm} 92.30 \%(72 / 78)$ and $>$ $10 \mathrm{~cm} \mathrm{90.90 \%} \mathrm{(10/11)} \mathrm{were} \mathrm{higher} \mathrm{than} \mathrm{that} \mathrm{in} \leqslant 2 \mathrm{~cm}$ HCC $50.0 \%$ (7/14), respectively. Similarly, the intratumoral Contactin expression significantly correlated with 


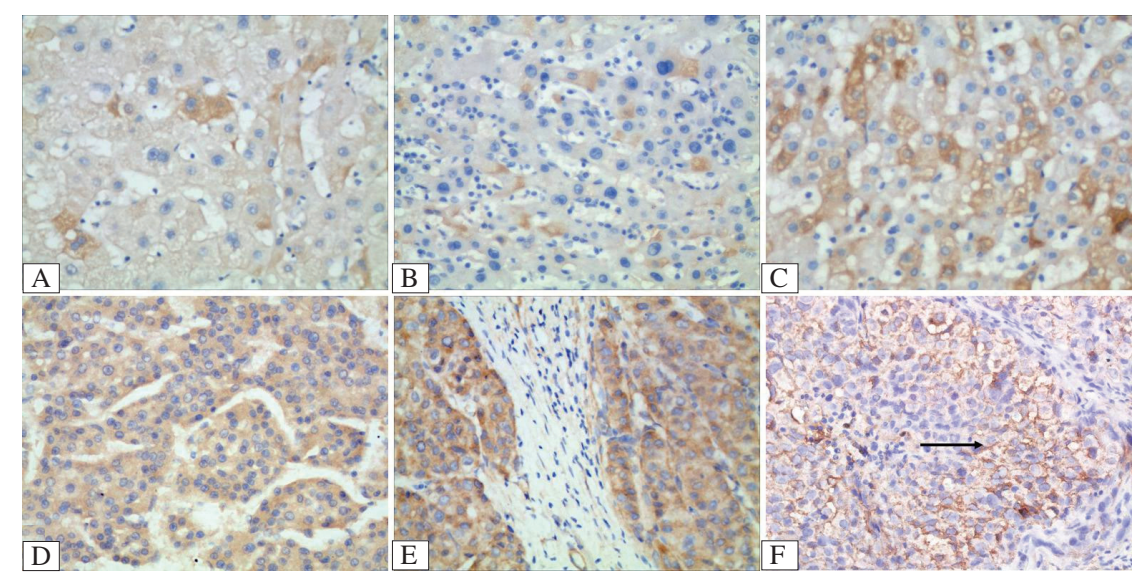

Fig.1. NET-1 expressed in (a) ANT, (b) well-differentiated HCC, (c) moderately differentiated HCC, (d) poorly differentiated HCC, (e) HCC with cirrhosis, (f) the marginal cells of the cancer nest (indicated by arrowheads), respectively. NET -1 expression was stronger in HCC than that in ANT $(\times 200)$.

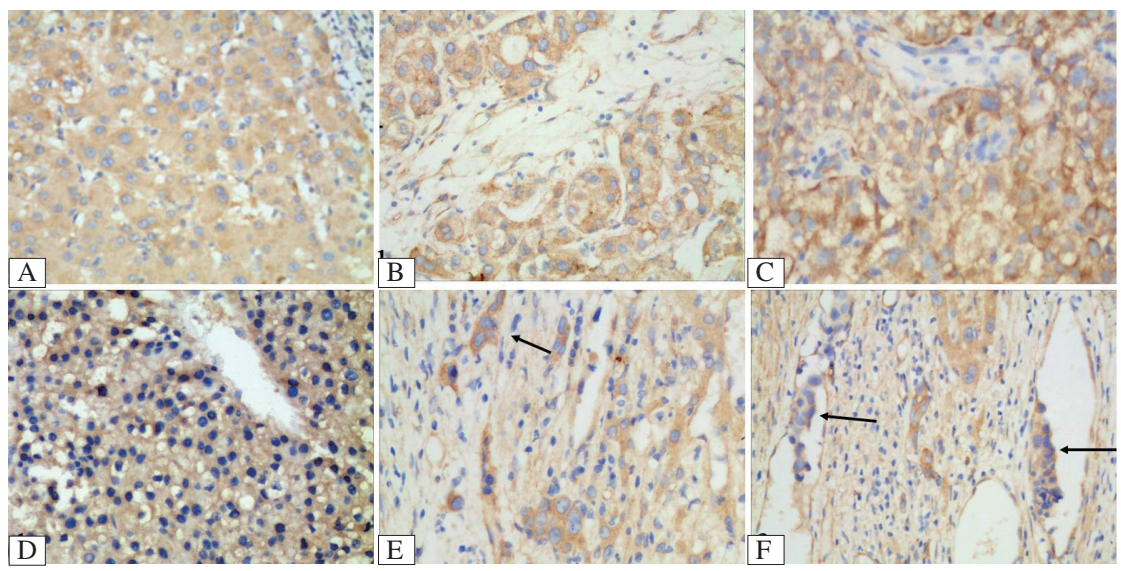

Fig.2. Cortactin expressed in ANT(a), well-differentiated HCC(b), moderately differentiated HCC(c), poorly differentiated HCC(d), HCC with stroma infiltration (e)indicated by arrowheads), HCC with vascular invasion (f)indicated by arrowheads) respectively. Contactin expressed in the cytoplasm of HCC cells, and the staining intensity increased with increasing grade of $\mathrm{HCC}(\times 200)$.

TNM stage $\left(\chi^{2}=22.200, P=0.001\right)$, vascular invasion $\left(\chi^{2}=15.185, \mathrm{P}=0.001\right)$ and capsular or tumor around infiltration $\left(\chi^{2}=8.532, P=0.014\right)$. The Contactin positive rate in TNM I - II stage in HCC with TNM III - IV stage $88.46 \%(46 / 52)$ was higher than that with I II stage $56.86 \%$ (29/51), in HCC with vascular invasion $90.91 \%$ (30/33) than that without vascular invasion $64.28 \%$ (45/70), in HCC with capsular or tumor around infiltration 88.89\% (32/36) was higher than that without infiltration $64.18 \%$ (43/67).

\section{The expression of NET-1 correlated with Cortactin in HCC}

The expression of NET -1 was found to be correlated with Contactin in HCC as shown in Table 5. Spearman related analysis showed the significant positive association between the expression of NET-1 and Contactin in 103 cases HCC patients $(r=0.280, P=0.004)$.

The correlation of NET-1 and Cortactin ex-

\section{pressions with prognosis of HCC}

The associations between either NET-1 or Contactin expression and patient survival were investigated using Kaplan-Meier analysis and log-rank test for singlefactor and multivariate analysis with 103 cases of HCC follow-up data. The results indicated that the median OS rates after operation for the 103 patients was 41 months (ranging from 1 to 91 months), the 1-, 3- and 5 -year OS rates were $81.6 \%$ (84 cases), $53.4 \%$ (55 cases) and $33.98 \%$ (35 cases), respectively.

The 5-year OS of patients with NET-1 negative expression $57.14 \%$ (8/14) were significantly higher than those with positive expression $31.46 \%$ (27/89), and the log-rank test demonstrated a significant difference $(P<0.05$, Fig. $3 \boldsymbol{A})$. The $5-y e a r$ OS of patients with Contactin negative expression $50.0 \%$ (14/28) were significantly higher than those of positive expression $28.0 \%(21 / 75)$, and the log-rank test demonstrated significant difference $(P=0.004$, Fig. $3 \boldsymbol{B})$. No significant 
Table 4 Correlations between either NET-1 or Cortactin expressions and clinicopathologic parameters in HCC (103 cases)

\begin{tabular}{|c|c|c|c|c|c|c|c|c|c|c|c|}
\hline \multirow{2}{*}{ clinicopathologic parameters } & \multicolumn{6}{|c|}{ NET-1 } & \multicolumn{5}{|c|}{ Cortactin } \\
\hline & $n$ & - & + & ++ & $x^{2}$ & $P$ & - & + & ++ & $x^{2}$ & $P$ \\
\hline Age (years) & & & & & 1.800 & 0.406 & & & & 1.160 & 0.560 \\
\hline$<50$ & 53 & 6 & 20 & 27 & & & 12 & 20 & 21 & & \\
\hline$\geqslant 50$ & 50 & 8 & 23 & 19 & & & 16 & 16 & 18 & & \\
\hline Sex & & & & & 0.281 & 0.869 & & & & 0.025 & 0.987 \\
\hline Male & 85 & 12 & 36 & 37 & & & 23 & 30 & 32 & & \\
\hline Female & 18 & 2 & 7 & 9 & & & 5 & 6 & 7 & & \\
\hline Edmondson's grade & & & & & 11.424 & 0.003 & & & & 2.750 & 0.253 \\
\hline I - II grade & 49 & 11 & 13 & 25 & & & 17 & 16 & 16 & & \\
\hline III - IV grade & 54 & 3 & 30 & 21 & & & 11 & 20 & 23 & & \\
\hline TNM stage & & & & & 16.434 & 0.012 & & & & 22.200 & 0.001 \\
\hline I & 10 & 5 & 2 & 3 & & & 8 & 1 & 1 & & \\
\hline II & 41 & 6 & 17 & 18 & & & 14 & 12 & 15 & & \\
\hline III & 36 & 2 & 19 & 15 & & & 5 & 15 & 16 & & \\
\hline IV & 16 & 1 & 5 & 10 & & & 1 & 8 & 7 & & \\
\hline Vascular invasion & & & & & 0.301 & 0.860 & & & & 15.185 & 0.001 \\
\hline Absence & 70 & 10 & 30 & 30 & & & 25 & 27 & 18 & & \\
\hline Presence & 33 & 4 & 13 & 16 & & & 3 & 9 & 21 & & \\
\hline Cirrhosis & & & & & 38.888 & 0.001 & & & & 3.772 & 0.152 \\
\hline No & 20 & 11 & 2 & 7 & & & 8 & 8 & 4 & & \\
\hline Yes & 83 & 3 & 41 & 39 & & & 20 & 28 & 35 & & \\
\hline AFP (ng/mL) & & & & & 1.958 & 0.376 & & & & 0.571 & 0.752 \\
\hline$<50$ & 39 & 6 & 19 & 14 & & & 9 & 14 & 16 & & \\
\hline$\geqslant 50$ & 64 & 8 & 24 & 32 & & & 19 & 22 & 23 & & \\
\hline HBsAg & & & & & 1.729 & 0.421 & & & & 4.824 & 0.090 \\
\hline Negative & 20 & 4 & 6 & 10 & & & 6 & 3 & 11 & & \\
\hline Positive & 83 & 10 & 37 & 36 & & & 22 & 33 & 28 & & \\
\hline Infiltration & & & & & 2.660 & 0.264 & & & & 8.532 & 0.014 \\
\hline No & 67 & 10 & 31 & 26 & & & 24 & 23 & 20 & & \\
\hline Yes & 36 & 4 & 12 & 20 & & & 4 & 13 & 19 & & \\
\hline Tumor nodules & & & & & 2.771 & 0.250 & & & & 3.294 & 0.193 \\
\hline Single & 72 & 9 & 27 & 36 & & & 16 & 28 & 28 & & \\
\hline Multiple & 31 & 5 & 16 & 10 & & & 12 & 8 & 11 & & \\
\hline Tumor size $(\mathrm{cm})$ & & & & & 19.370 & 0.001 & & & & 4.135 & 0.388 \\
\hline$\leqslant 2$ & 14 & 7 & 5 & 2 & & & 4 & 4 & 6 & & \\
\hline $2-10$ & 78 & 6 & 33 & 39 & & & 22 & 30 & 26 & & \\
\hline$>10$ & 11 & 1 & 5 & 5 & & & 2 & 2 & 7 & & \\
\hline
\end{tabular}

Table 5 Correlation between the expression of NET-1 and Cortactin in HCC

\begin{tabular}{ccccc}
\hline \multirow{2}{*}{ expression } & & \multicolumn{3}{c}{ Cortactin } \\
\cline { 3 - 5 } & & - & + & + \\
\hline NET-1 & - & 11 & 2 & 1 \\
& + & 9 & 16 & 18 \\
& ++ & 8 & 18 & 20 \\
\hline
\end{tabular}

difference on 5-year OS was observed between lower expression (+) group and intensive expression (++) group of either NET-1 or Contactin $(P>0.05)$, so the two groups are merged into one group.
The association of the co-expression of NET-1 and Contactin with OS was investigated further. All the cases were divided into four groups according to the expression of NET-1 and Contactin. The 5-year OS of patients were investigated by using Kaplan-Meier analysis (Fig. 3C) and log-rank test (Table 6). The results indicated that the 5-year OS of patients with positive co-expression of NET-1 and Contactin genes (27.78\%) were significantly lower than that with negative expression of both genes $(54.54 \%)$, and NET-1 positive with Contactin negative $(47.06 \%$ ), both $P<0.05$; the $5-$ year OS of patients with positive Contactin and negative NET-1 ex- 

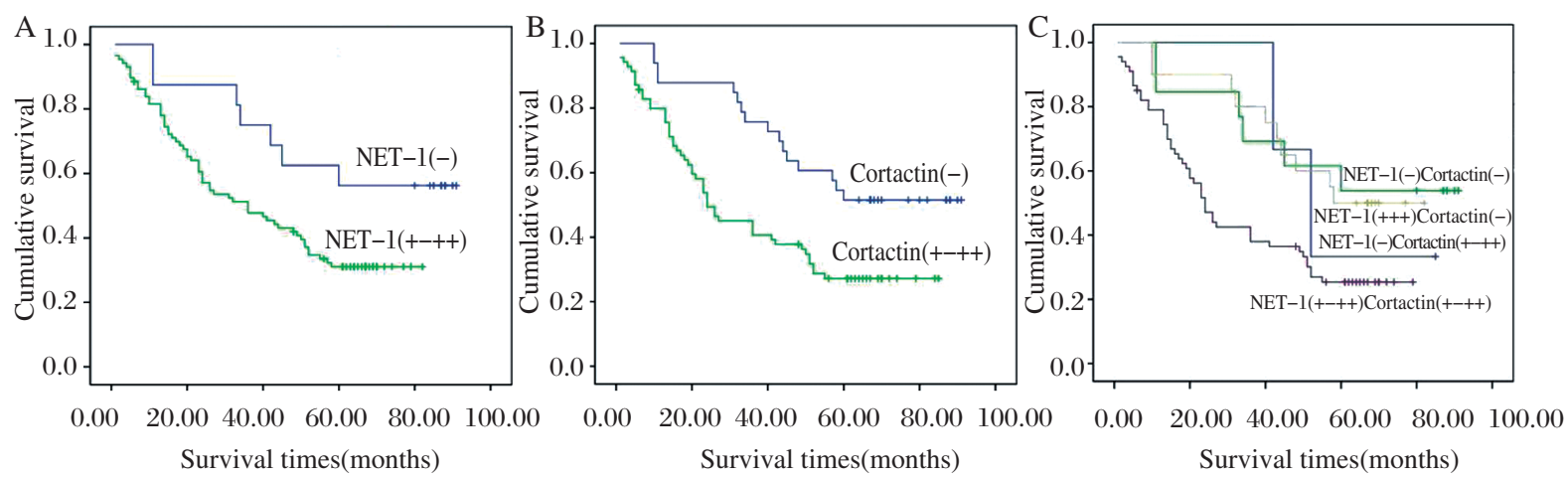

Fig.3. Kaplan-Meier survival curves of HCC, stratified for NET-1 expression in the whole study population. It was showed that the median OS of NET-1 positive group was much lower than that of negative groups (A), the median OS of Contactin positive group was much lower than that of negative groups (B), and the median OS of patients with positive co-expression of NET-1 and cortactin genes was much lower than that with negative expression of both genes $(\mathrm{C})(\log -$ rank test, $P<0.05)$.

Table 6 The 5-year OS of patients with NET and Contactin expression

\begin{tabular}{clccccc}
\hline Groups & Gene expression & Cases & Survival & 5-year OS (\%) & Compared & Log-rank test $P$ \\
\hline (1) & NET-(1)-/Cortactin(+-++) & 3 & 1 & 33.30 & & \\
$(2)$ & NET-(1)-/Cortactin(-) & 11 & 6 & 54.54 & (1) vs. (2) & $<0.05$ \\
$(3)$ & NET-1(+-++)/Cortactin(+-++) & 72 & 20 & 27.78 & (2) vs. (3) & $<0.05$ \\
(4) & NET-1(+-++)/Cortactin(-) & 17 & 8 & 47.06 & (3) vs. (4) & $<0.05$ \\
\hline
\end{tabular}

pression was significantly lower than that with negative expression of both genes (33.3\% vs. 54.54\%, P<0.05). The 5-year OS of patients with positive co-expression of NET -1 and Contactin genes was significantly lower than that with positive Contactin and negative NET -1 expres$\operatorname{sion}(27.78 \%$ vs. $33.3 \% P<0.05)$.

Cox regression analysis was used to compare the
NET-1 and Contactin expressions with clinicopathological features for survival prediction. As shown in Table 7, by univariate Cox regression analysis, the parameters such as late stage, large tumor size, NET-1 and Contactin over expressions, vascular invasion and capsular or tumors around tissue infiltration, were significantly associated with poor OS. By multivariate Cox regression

Table 7 Cox regression analysis for overall survival based on NET-1 and Contactin over expressions and clinicopathological variables

\begin{tabular}{|c|c|c|c|c|}
\hline \multirow{2}{*}{ Variables } & \multicolumn{2}{|c|}{ Univariate analysis } & \multicolumn{2}{|l|}{ Multivariate analysis } \\
\hline & Hazard ratio $(95 \% \mathrm{CI})$ & $P$ & Adjusted Hazard ratio (95\% CI) & $P$ \\
\hline Age & $0.814(0.500-1.325)$ & 0.407 & & \\
\hline Sex & $1.139(0.621-2.091)$ & 0.673 & & \\
\hline Edmondson's grade & $0.936(0.648-1.351)$ & 0.724 & & \\
\hline TNM staging & $1.894(1.405-2.555)$ & $<0.001$ & $1.948(1.410-2.692)$ & $<0.001$ \\
\hline Vascular invasion & $1.945(1.174-3.222)$ & 0.010 & & 0.999 \\
\hline Serum AFP level & $1.606(0.961-2.683)$ & 0.071 & & \\
\hline HbsAg status & $1.317(0.689-2.516)$ & 0.405 & & \\
\hline Infiltration & $0.555(0.340-0.906)$ & 0.018 & & 0.896 \\
\hline multinodular tumor & $0.998(0.521-1.909)$ & 0.995 & & \\
\hline Tumor sizes & $2.228(1.353-3.669)$ & 0.002 & $2.459(1.158-5.226)$ & 0.019 \\
\hline NET-1 expression & $3.175(1.361-7.403)$ & 0.007 & $3.573(2.033-6.082)$ & $<0.001$ \\
\hline
\end{tabular}

analysis, only TNM stage, tumor size, NET-1 and Contactin over expression were identified to be independent prognostic factors for OS $(P<0.05)$. It was illustrated that NET -1 and Cortactin, together with tumor size and TNM stages, were strongly associated with OS. However, other variables including age, sex, Edmondson's grade, cirrhosis background, serum AFP level, HBsAg status and multinodular tumor seemed to show a general trend with subtle differences in survival without significance.

\section{The expression of NET-1 and Contactin in hu- man HCC cells}

The NET-1 and Cortactin protein levels in SMMC7721, Huh7 and HepG2 cells were detected by Western 
blot (Fig. 4). The results showed that the protein level of NET-1 and Cortactin were higher in SMMC-7721 than that in Huh7 or HepG2 cells, thus SMMC-7721 were selected for following experiments.

\section{The expression of NET-1 and/or Contactin downregulated by siRNA}

The mRNA and protein levels of NET-1 and Cortactin in SMMC-7721 cells transfected with single

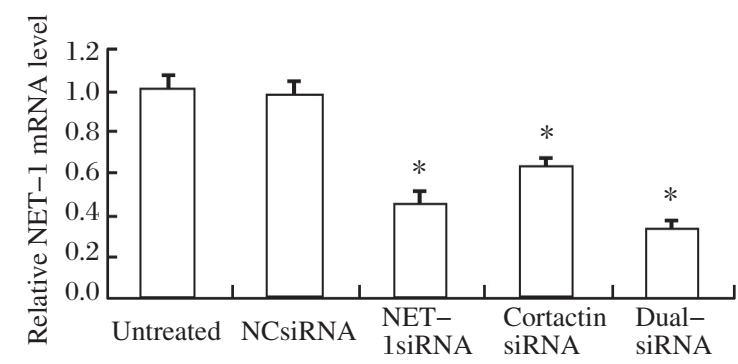

B

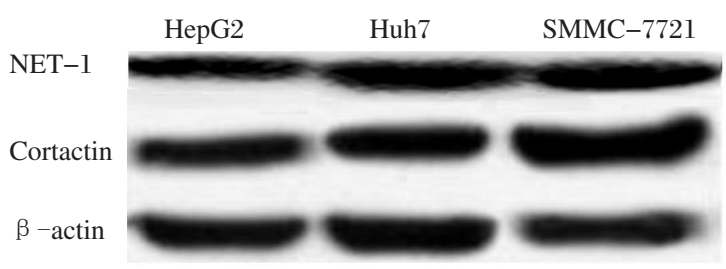

Fig. 4. NET-1 and Cortactin protein levels in SMMC-7721, Huh7 and HepG2 cells (detected by Western blot)
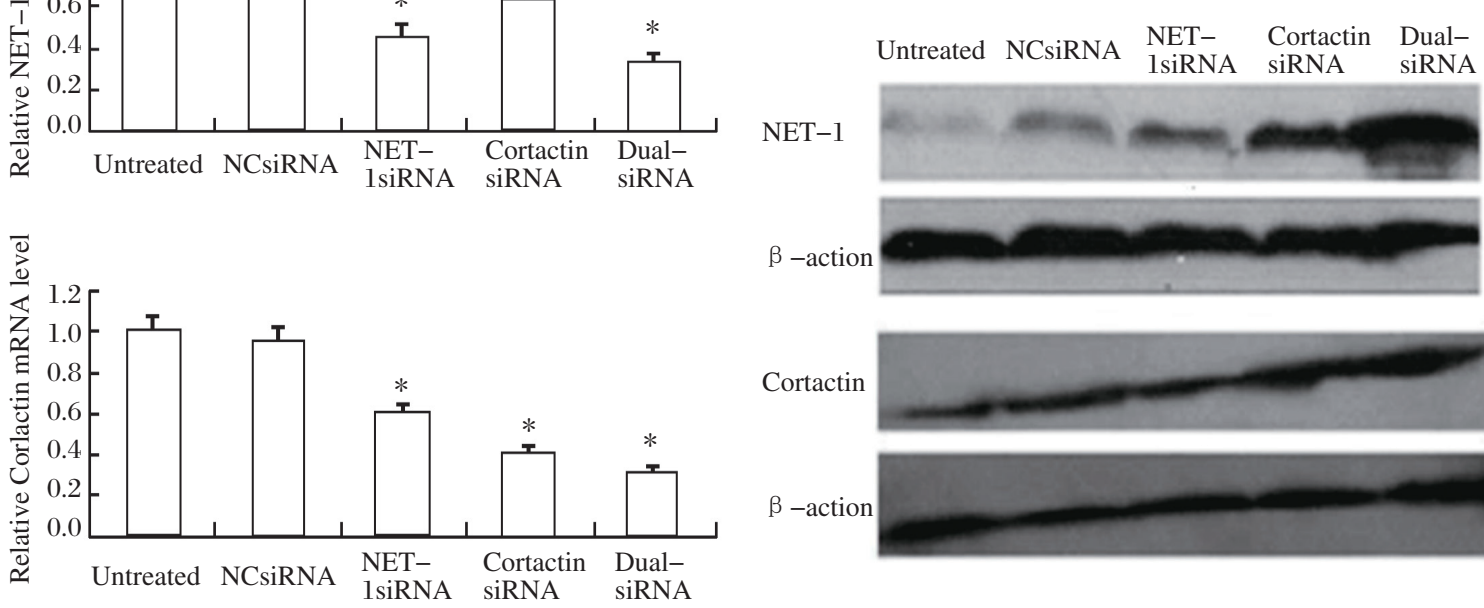

Cortactin

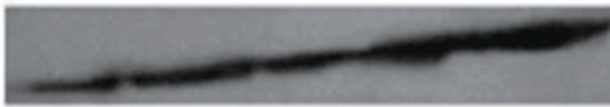

$\beta$-action

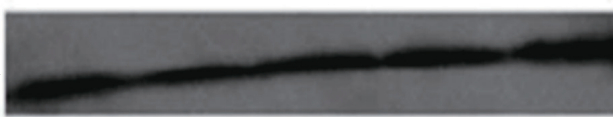

C

NET-1
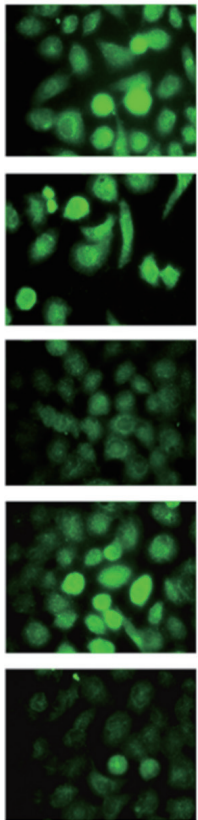

Hoechest
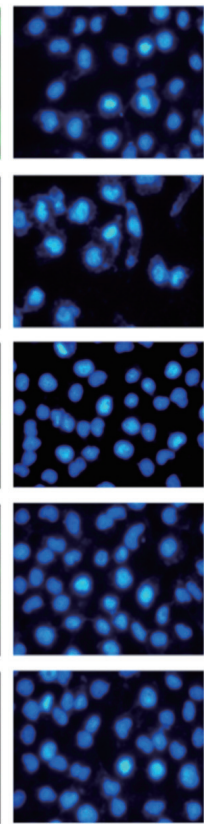

Merged
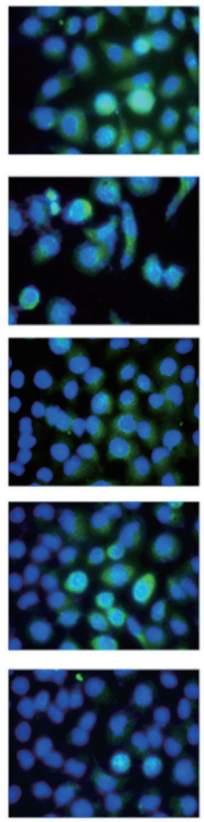
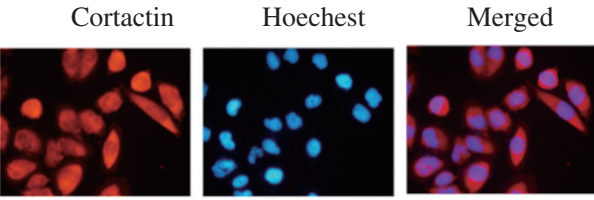

Untreated
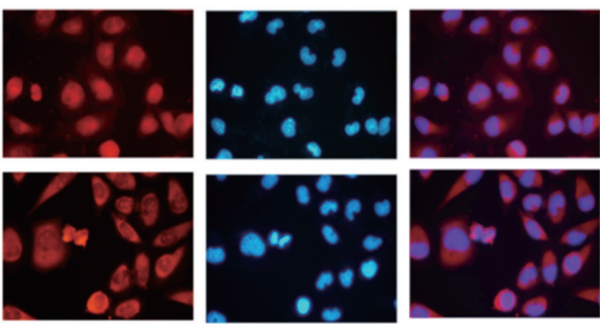

NC siRNA
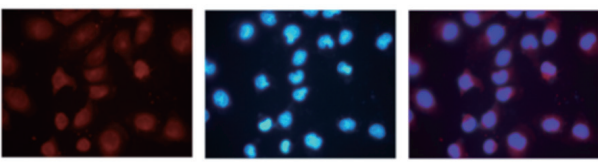

Cortactin siRNA
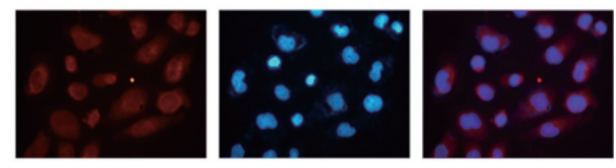

NET-1 siRNA

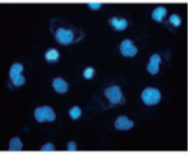

Dual-siRNA

Fig.5. The expression of NET-1 and Cortactin mRNA and protein in SMMC-7721 cells. A: The mRNA levels of NET-1 and Cortactin in SMMC-7721 cells treated with siRNAs detected by RT-qPCR. " $P<0.05$ vs. untreated cells; B: The protein levels of NET-1 and Cortactn in SMMC7721 cells inhibited treated with siRNAs detected by Western blot. C: The expression of NET-1 and Cortactn treated with different siRNAs detected by immunofluorescence staining $(\times 200)$. NET-1 (green) and Cortactin (red), the nuclei of cells were stained by Hoechst in blue. 
target siRNAs or dual-siRNA were detected by RTqPCR and Western blot.

Compare with untreated cells, NET-1 or Cortactin were reduced by NET-1 siRNA or Cortactin siRNA up to $45 \%$ and $39 \%$, respectively, while dual-siRNA suppressed both genes by $33 \%$ (NET-1) and 30\% (Coractin) simultaneously on mRNA level detected by RT-qPCR(Fig. 5A). Futhermore, NET-1 or Cortactin were decreased by NET-1 siRNA or Cortactin siRNA up to $64.67 \%$ or $63.61 \%$, while NET-1 and Coractin were suppressed by dual-siRNA up to $30.44 \%$ and $49.15 \%$ on protein level detected by Western blot $(P<0.05)$ (Fig. 5B). The same results were observed using immunofluorescent microscopy (Fig.5C).

\section{The proliferation of HCC cells inhibited by siRNAs}

CCK-8 assay was performed to compare the growth rate of cells in different groups. After treated with siRNAs, the growth of HCC cells were all inhibited by single-target siRNAs or dual-siRNA $(P<0.05)$, compared with untreated cells. The effect of dual-siRNA was the strongest $(P<0.01)$. There was no statistical difference in both single-target siRNA and between untreated cells and NC siRNA treated cells(Fig. 6).

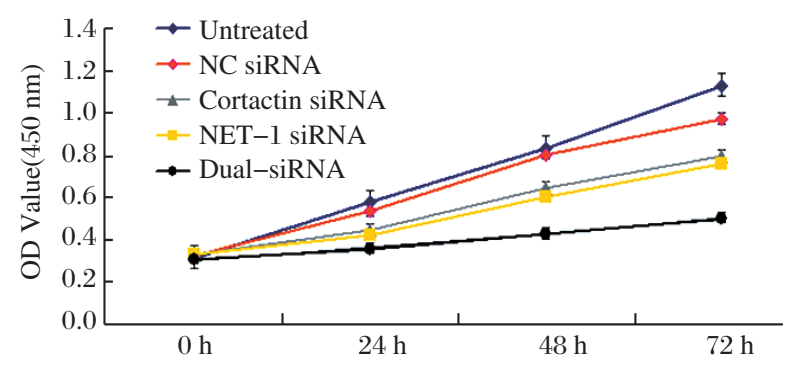

Fig.6. The growth curves of SMMC-7721 cells treated with siRNAs using CCK-8 assay. OD values were presented at the indicated time points as the mean absorbance with a standard deviation of 3 wells. The inhibiting effects on the proliferation of SMMC-7721 were orderly as compared with untreated cells, as was dual-siRNA, NET-1 siRNA and Cortactin siRNA. There was no statistical difference in both single target siRNA and between untreated group and NC siRNA.

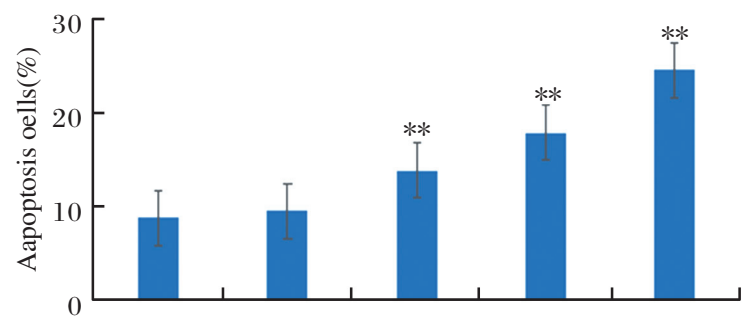

Fig. 7. Cell apoptosis of SMMC-7721 cells treated with siRNAs analysis by FCM (Histogram, ${ }^{* *} P<0.05$ vs. untreated).

\section{The apoptosis of HCC cells induced by siRNAs}

The percentage of apoptosis cells was determined by FCM. Compared with untreated cells $(8.75 \% \pm 0.39 \%)$ and NC siRNA $(9.43 \% \pm 1.02 \%)$, the apoptosis rate of HCC cells were increased when treated by Cortactin siRNA $(13.85 \% \pm 0.95 \%)$, NET -1 siRNA $(17.89 \% \pm 1.01 \%)$ and dual-siRNA $(24.64 \% \pm 0.83 \%)$ (Fig. 7). The two-sample $t$ test with equal variance analysis showed a statistical significance between each experimental group and untreated group, respectively(every $P<0.001$ ). The promoting effect of apoptosis in dual-siRNA group was significantly higher than either single target group.

\section{The migration of HCC cells inhibited by siRNAs}

The wound healing assay and transwell chamber assay were used to assess whether the migration ability of SMMC-7721 cells was affected by NET -1 and Cortactin.

The result of the wound healing assay showed the scratched lanes were covered by migrating cells $72 \mathrm{~h}$ later in untreated and NC siRNA treated cells. There was no significant difference between untreated and NC siRNA treated cells $(P>0.05)$. In contrast, the scratched lanes were not completely occupied by both single-target siRNAs and dual-siRNA treated cells(Fig. $8 \boldsymbol{A}$ ).

In transwell assay, compare with untreated (164.03 \pm 34.04$)$ and NC siRNA (158.67 \pm 26.11$)$ treated cells, the invasion abilities of HCC cells were inhibited by Cortactin siRNA (111.19 \pm 32.51$)$, NET-1 siRNA (140.24 \pm 25.78$)$ and dual-siRNA (84.11 11.34$)$, respectively(Fig. $8 B$ ).

\section{The growth of xenograft tumors inhibited by siRNAs}

SMCC-7721 cells were inoculated into nude mice subcutaneously and allowed to develop xenograft tumors in vivo, and 14 days later, cationic lipids encapsulated dual-siRNA or negative control (NC siRNA) were injected into tumors, as PBS as a untreated control. After another 16 days, the volume and weight of tumors in dual-siRNA treated group $[(320.1 \pm 8.4)$ $\mathrm{mm} 3$ and $(0.80 \pm 0.12) \mathrm{g}]$, NC siRNA treated group $\left[(744.3 \pm 11.6) \mathrm{mm}^{3}\right.$ and $\left.(1.79 \pm 0.19) \mathrm{g}\right]$ and untreated group $\left[(856.2 \pm 19.4) \mathrm{mm}^{3}\right.$ and $(1.90 \pm 0.21) \mathrm{g}$ ] were measured, dual-siRNA showed a greater impact on tumor growth than NC siRNA and PBS treatment $(P<0.01$, Fig. 9A).

Tumors were harvested from animals when the experiment finished, and sections were stained with $H \& E$ for morphological observation. H\&E staining showed the increased nuclear mitosis with large area necrosis 
A

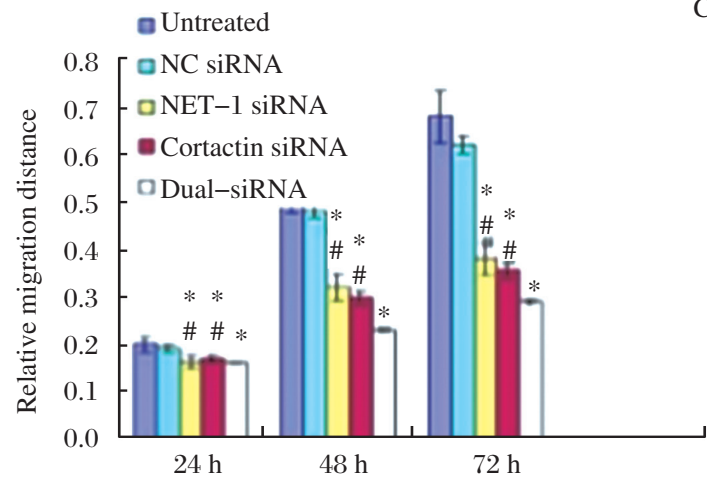

B

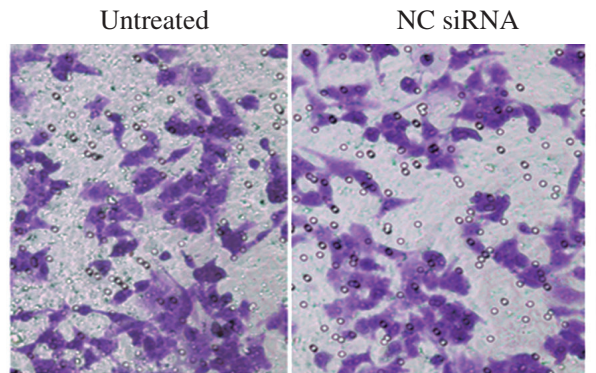

C

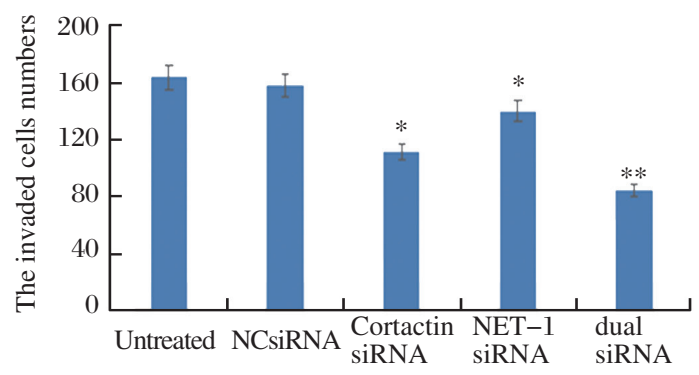

Cortactin siRNA
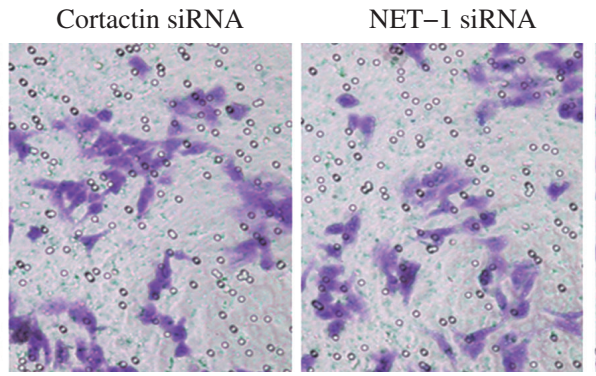

Dual-siRNA

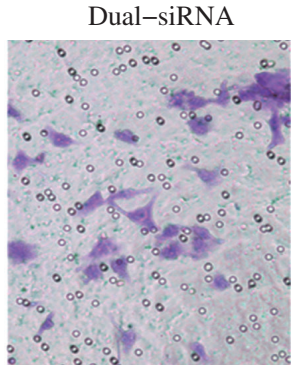

Fig. 8. Effects of siRNAs targeted NET-1 and/or Cortactin on SMMC-7721 cell migration. A: Histogram of relative migration distances 24,48 and $72 \mathrm{~h}$ after treated with siRNAs. ${ }^{*} P<0.05 v$ s. untreated cells, ${ }^{\sharp} P<0.05 v s$. untreated cells; B: The invaded cells in the transwell assay $48 \mathrm{~h}$ after transfection. The invading cells on the underside of the filter were stained with crystal violet $(\times 200)$. C: Histogram ${ }^{*} P<0.05$ vs untreated cells, ${ }^{* *} P<0.01$ vs untreated cells.
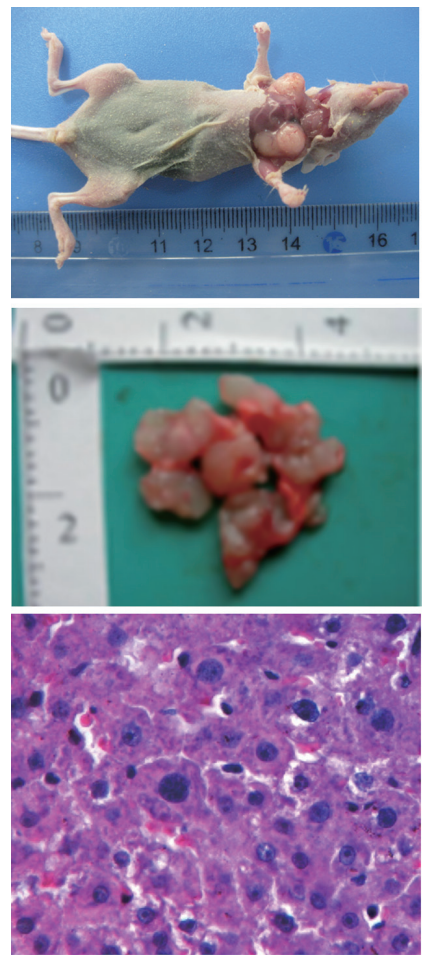

Untreated
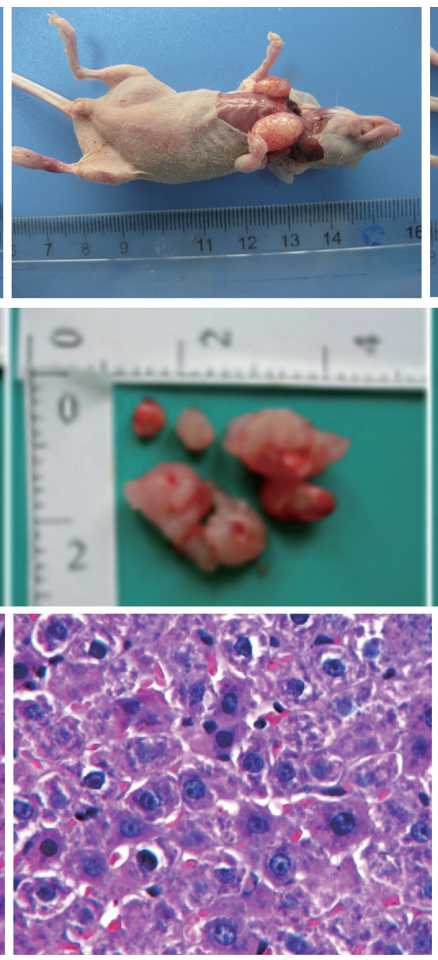

NCsiRNA

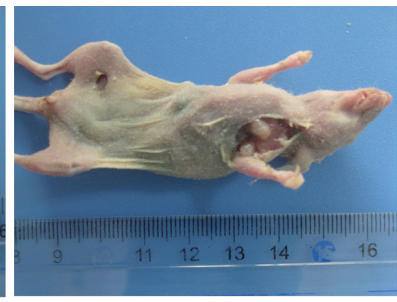

Nude mice model
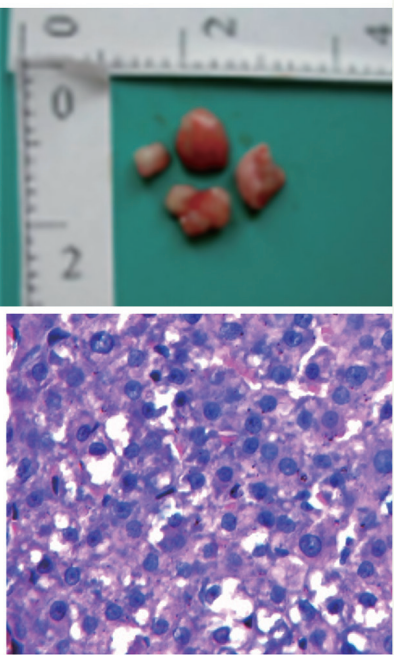

Dual-siRNA

Fig.9. Pathological observations in nude mice xenograft tumors. In the gross manifestation of nude mice xenograft tumors, the size of the tumor in the dual-siRNA treated group was significantly smaller than that in the NCsiRNA and PBS treated groups. In high power view, the increased nuclear mitosis was in the NCsiRNA and PBS treated groups (indicated by arrow), whereas the increased cell apoptosis was observed in dual-siRNA treated group $(\times 400)$. 
in the center of the tumor in the groups treated with NC siRNA and PBS, whereas the dual-siRNA treated group showed less nuclear mitosis with increasing cell apoptosis (Fig. 9C).

Xenograft tumor sections were stained with IHC for detecting the expression of NET-1, Cortactin, Ki67, Bax, Bcl-2 and Survivin (Fig. 10), the cell numbers with positive immunoreactivity were obtained in 5 randomly microscopic fields in a section $(n=5)$. The cell numbers positive with NET-1, Cortactin, Ki67, Sur- vivin and Bcl-2 were significantly reduced, while the cells positive with Bax were significantly increased in dual-siRNA treated group compared with NC siRNA and PBS treated groups (every $P<0.05$ ).

\section{DISCUSSION}

NET -1 and Cortactin have been previously reported as cancer-associated genes. Current evidences suggest that they play important roles in up-regulating carcino-

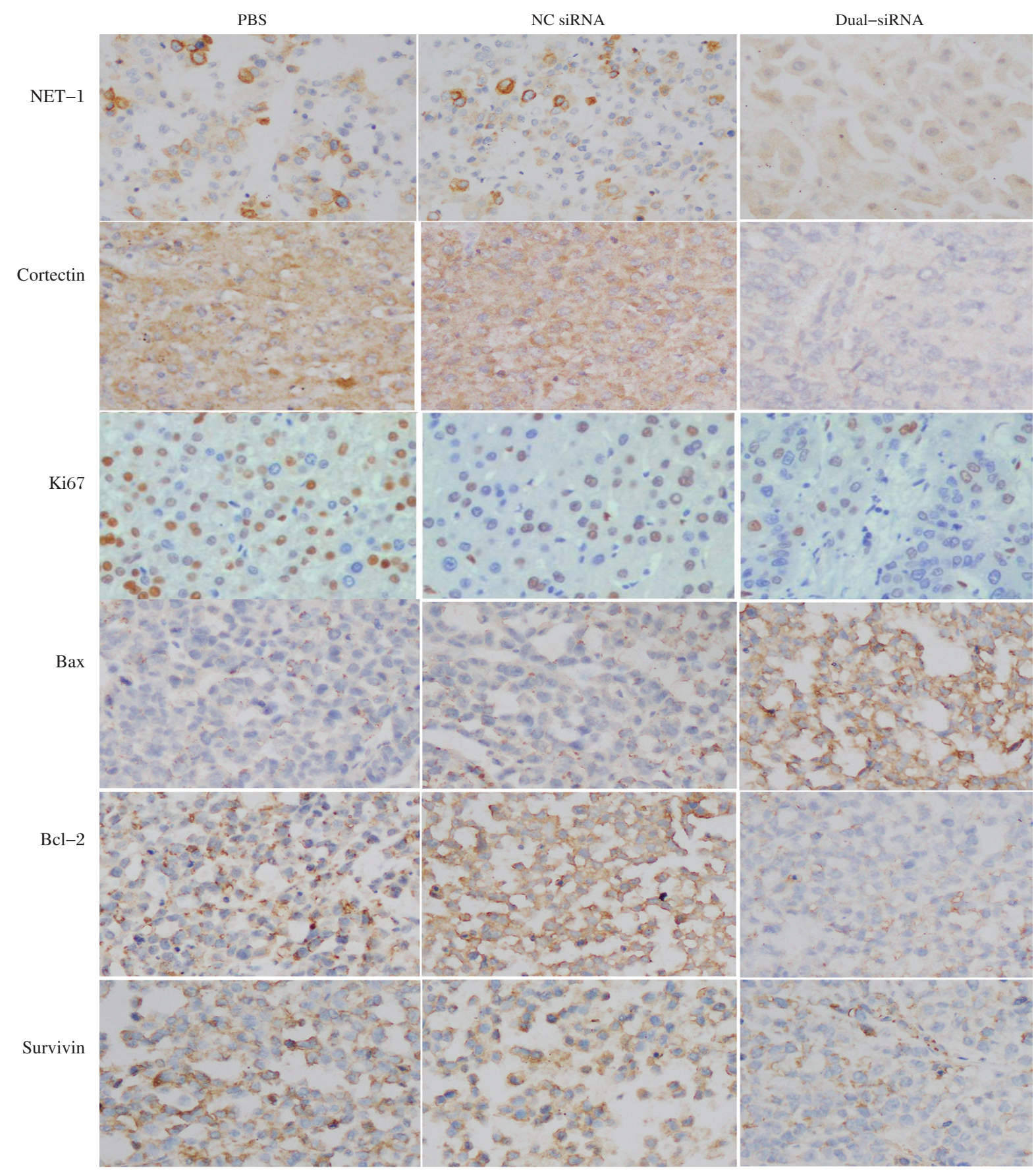

Fig.10. The expression of NET-1, Cortactin, Ki67, Bax, Bcl-2 and Survivin in nude mouse xenograft tumor tissues. The cells positive with NET-1, Cortactin, Ki67, Survivin and Bcl-2 were significantly reduced, while Bax positive cells were significantly increased in dual-siRNA treated tumors compared with NC siRNA and PBS treated tumors $(\mathrm{Ki} 67, \times 400$; the remaining slices $\times 200)$. 
genesis and are closely related to cancer prognosis ${ }^{[12-21]}$.

NET-1 acts as the molecular service protein, which enhances the formation and stability of the functional signal transduction complex by connecting specific cell surface proteins such as lineage specific proteins, integration proteins and other TM4SFs members. So it plays an important role in cell signal transduction, adhesion, migration, proliferation and differentiation ${ }^{[5-6]}$. Previous studies have shown that NET-1 expression was closely related with cell proliferation, as a result, it promoted the formation of cancers significantly ${ }^{[16-23]}$. Our results confirmed that NET-1 expression in HCC (85.96\%) is significantly higher than in adjacent nontumor tissues (59.6\%). In HCC, the expressive intensity of NET-1 is positively correlated with pathological Edmondson's grade and the clinical TNM stage. NET -1 expressions in larger tumors and in the marginal cells of cancer nests are higher than those in smaller tumors and in the center of cancer nests, in HCC with cirrhosis are higher than those without cirrhosis. These results may be due to the uncontrolled proliferation and poorer differentiation of tumor cells, similarly, the cells in the margin of cancer nests often are actively proliferating, whereas the cells in the center of cancer nests with hypoxia, hypoemia and lower nurture were easily induced cell death. Cirrhosis background stimulates cancer cell proliferation and provides the environment of cell transformation. In vitro, $\mathrm{CCK}-8$ assay showed the proliferation of SMCC-7721 cells was inhibited by the down-regulated NET-1 gene, which also promoted the cell apoptosis of SMCC-7721. It is suggested that NET-1 acts as a tumor promoter and its over expression directly effects hepatocarcinogenesis by transecting the cell proliferating signal to accelerate cell malignant transformation, which may partly explain the up-regulation of NET-1 expression in $\mathrm{HCC}^{[16-18,27]}$. Consequently, prognostic analysis showed that the 5-year overall survival rate of patients with NET-1 positive expression was significantly lower than that with negative expression. Thus the NET-1 expression may serve as a potential factor for influencing prognosis of $\mathrm{HCC}^{[17-18]}$.

Cortactin (cortical actin-binding protein) can bind to the Arp2/3 complex in cytoplasm and activate actin polymerization mediated by the Arp $2 / 3$ complex to promote cell migration ${ }^{[7-11]}$. The research of Kowalski JR et al. ${ }^{[28]}$ confirmed that this effect was carried out by binding and activating neural Wiskott Aldrich Syndrome protein (N-WASP), thus promoted Arp2/3 actin nucleation and cell migration successively. Our study shows that Contactin expression in HCC tissues (73.68\%) is significantly higher than that in adjacent nontumor tissues (28.1\%); it indicates that Contactin is a cancer-asso- ciated gene. In HCC, Contactin is strongly expressed in the tissues with diffusely infiltrated stroma, vessels and capsular, and significantly correlates with TNM stages of HCC. The results of transwell chamber assay and wound healing assay show the migration of SMCC-7721 cells were inhibited by the down-regulated Contactin gene in vitro. Conversely, the up-regulation of Contactin expression in $\mathrm{HCC}$ is significantly associated with cancer cells infiltration and invasive activity ${ }^{[7-9]}$. Our analyses on the following-up data from 103 patients further confirmed that high expression of Contactin promoted the malignant progression of HCC and related to poor prognosis $^{[12-13]}$.

As tumor associated genes, the expression of NET-1 and Contactin of HCC is closely related with cell proliferation and makes the cells more actively invasive. Though several authors have described the expression of NET-1 and Contactin in $\mathrm{HCC}^{[12-13,16-18]}$, combined actions for NET-1 and Contactin in HCC have not been reported before, and their potential roles on the development and survival rate of HCC need to be fully characterized. Therefore, we hypothesized that the coexpression of both genes in HCC has a synergistic effect. Focusing on the significance and function of both genes, this paper revealed the positive relationship between NET-1 and Contactin expression in HCC, and demonstrated that the co-expression of both genes is associated with HCC malignant progression, which reduce 5-year OS of patients who underwent curative resection for HCC to $27.78 \%$, it's significantly less than the other expressing conditions, such as NET-1 negative with Contactin positive (33.33\%), NET-1 positive with Contactin negative (47.06\%), and both genes negative (54.54\%), respectively.

Some of the clinicopathological factors (TNM stage, tumor size, vascular, capsular or stroma infiltration) and the expressions of NET-1 or Contactin were screened out by univariate Cox regression analysis for their effects on survival and poor prognosis of HCC. However, by multivariate Cox regression analysis, only TNM stage, larger tumors, NET-1 or Contactin expressions are suggested to be independent prognostic factors for OS. Other factors, such as age, sex, Edmondson's grade, cirrhosis background, serum AFP level, HBsAg status and multinodular tumor, show some effects on survival without significance. The results also indirectly supports the concept that synergistic effects of NET-1 and Contactin may contribute to tumor progression and poor prognosis by simultaneously promoted the proliferation, invasion and metastasis of HCC.

Because of efficient and specific silencing of target protein expression, siRNA has recently been experimentally used to treat cancers. However, the efficiency 
of siRNA targeting a single gene is limited. As for anticancer therapy, inhibition of a single oncogenic gene is also insufficient to kill tumor cells, because oncogenesis is correlated with multiple genes involved in multiple pathways ${ }^{[29-30]}$. Therefore, developing multi-target siRNAs could be an advantageous strategy, by which multiple gene expressions can be inhibited simultaneously and thus it may lead to more effective anticancer therapies than what single-target siRNA could achieve. In addition to multi-cassette shRNAs ${ }^{[31]}$ and long hairpin shRNAs ${ }^{[32]}$, studies on synthetic multi-target siRNAs have also been reported. Hossbach $\mathrm{M}$ et al. ${ }^{[33]}$ reported a dual-targeting siRNA composed of two fully targetcomplementary guide strands, with the strands partially complementary to each other. An algorithm was further developed to predict dual-targeting siRNAs with complete complementary strands which acted as active guide strands separately targeting different mRNAs ${ }^{[34]}$.

To further confirm the synergistic effects of NET1 and Cortactin, we postulated if the knockdown of both NET -1 and Cortactin genes would eliminate their influence on the progression and metastasis of HCC. We firstly designed a new, long, simultaneously targeting NET-1 and Cortactin, double-stranded multiplex siRNA (dual-siRNA) structure composed of an intact long strand (4l nucleotides in length) and two complementary shorter strands (2l nucleotides in length). A gap between the two shorter strands also existed which resulted in the segmentation of the complementary strand. We simultaneously constructed single target siRNA against every gene (NET-1 siRNA and Cortactin siRNA), then transfected these siRNAs into HCC cells with higher expression of NET-1 and Contactin (SMMC-7721). The results proved that the dualsiRNA and single target siRNAs were all capable of silencing target mRNAs and protein expressions. Furthermore, they could significantly suppress proliferation and migration and induce apoptosis in SMCC-7721 cells. As compared to single target siRNAs, dual-siRNA was significantly more effective on inhibiting the biological behavior of HCC cells. This might be caused by the cytocidal effect while NET-1 and Contactin genes were simultaneously silenced by RNA interference in a way of inhibit cell proliferation and migration through the phosphoinositide of NET-1 and the unique mechanism of Contactin ${ }^{[5,28,35]}$. Our study revealed that RNA interference of NET-1 or Cortactin genes individually resulted in a small decrease in cell growth and spontaneously a modest increase in cell apoptosis. In contrast, when the suppression of NET-1 and Cortactin was achieved at the same time by dual-siRNA, the proliferation and migration of SMCC-7721 cells are dramatically inhibited and the apoptosis is induced. The gap in antisense strands of dual-siRNA is essential for the silencing activities of dual-siRNA, and the knockdown potency of dual-siRNA depends on the specific structures to a certain extent. It is well known that long dsRNA in cells will be digested by a dsRNA-specific endonuclease, Dicer, into short interfering RNA (siRNA) duplexes as functional effectors to cleave target mRNAs. Therefore, the gap between the two complementary strands may provide recognizable sites and/ or facilitate the process of Dicer, thus the resultant two siRNAs would then silence their targets, respectively. Based on the results above, we draw a conclusion that, when used alone dual-siRNA was demonstrated to have a higher effectiveness in inhibiting cancer cell growth than single-target siRNAs.

In vivo experiments, the dual-siRNA treatment group showed the smallest xenograft tumor volume and weight as compared with NC siRNA and PBS treated group. Tumor sections stained with H\&E showed increased nuclear mitosis with a large area of necrosis in the center of tumor in the groups treated with NCsiRNA and PBS treated group, whereas the less nuclear mitosis with increasing cell apoptosis was found in dual-siRNA treated group. The over-expressions of proliferation markers, such as Ki67, and anti-apoptotic signaling protein, such as Bcl-2, Bax and Survivin, have been shown to play a critical role in tumorgenesis ${ }^{[36-37]}$. Ki67 is a nuclear antigen expressed in proliferating cells in all stages of the cell cycle except stage G0 and serves as a marker for proliferation. $\mathrm{Bcl}-2$ is one of the main anti-apoptotic proteins normally residing in the mitochondrial membrane and cytoplasm. When cells are deprived of survival signals or subjected to stress, Bcl-2 is released from the mitochondrial membrane and replaced by proapoptotic factors, such as Bax. When the Bcl-2 levels decrease, the permeability of the mitochondrial membrane increases, and several proteins that can activate the caspase cascade are released ${ }^{[38]}$. To examine the underlying mechanisms of how NET-1 and Contactin affect HCC malignant transformation, we detected the expression of NET-1, Contactin , Ki67, Bcl-2, Bax and Survivin in xenograft tumor tissue of nude mice by immunohistochemistry stain. In the present study, NET-1, Contactin, Ki67, Survivin and Bcl-2 positive cells are significantly reduced, whereas Bax positive cells are significantly increased in dual-siRNA treated tumors compared with NC siRNA and PBS treated tumors. These observations revealed when the suppression of NET-1 and Cortactin was achieved at the same time by dual-siRNA, the growth of tumors was dramatically inhibited and the apoptosis were enhanced by a significant activation of Bax and downregulating of Ki67, Survivin and Bcl-2. It is indicated 
that both NET-1 and Cortactin genes have a capability to promote proliferation and inhibit cell apoptosis, a novel function involving a synergistic role was identified for both genes to participate in HCC progression. These provide possible explanations for the correlation between co-expression of NET-1 and Cortactin and poor prognosis in human HCC. In this work, the use of the novel, long dual-siRNA suggest that gene silencing siRNA structures are more flexible than originally proposed ${ }^{[39]}$, and are suitable for multiple gene silencing. Therefore, it could be anticipated that dual-siRNA can be applied in anticancer therapy for the genetic variability often results in an escape from a single RNAi trigger.

In conclusion, this paper confirmed a synergistic effect between NET-1 and Contactin in HCC progressive process, where the co-expression of both genes correlate to poor prognosis of HCC patients. Therefore, combined detection of NET-1 and Cortactin may have an important value in predicting the prognosis of $\mathrm{HCC}$ patients in clinical practice. Dual-siRNA exhibits more significant effect on the down-regulating of both gene mRNAs and proteins as well as suppressing the biological behavior of HCC cells in vitro and in vivo than either of single-target siRNA. We demonstrated here that dual-siRNA could be potentially utilized for simultaneously multiple gene silencing and the gap in the anti-sense strand is necessary to knock-down multiple genes. The designed dual-siRNA targeting NET-1 and Cortactin gene inhibits the growth of SMCC-7721 cells dramatically as compared with single individual targeting siRNAs. Therefore, dual-siRNA targeting NET1 and Contactin may provide a novel strategy for the treatment of HCC. This new type of dual-siRNA also has a great potential in other siRNA-based therapy, and further development of multiple-targeting RNA structures could still be worthwhile.

\section{Acknowledgements}

This study is supported by the foundation for Production-Study-Research Prospective Joint Research Programs of Jiangsu Province, China (No. 201304206), a Project Funded by the Priority Academic Program Development of Jiangsu Higher Education Institutions, Foundation of the Science Foundation of Nantong City, Jiangsu Province, China (No. HS2012070). And the Postgraduation Advanced Project Program of Nantong University (No.2013-29).

The authors are grateful to Affiliated Tumor Hospital and Affiliated Hospital of Nantong University for kindly helping to prepare tissue microarray. As well, thanks to Biomics Biotechnologies Co., Ltd for kindly construct the transmission system encapsulated cationic liposome for delivering dual-siRNA into HCC xenografts of mice.

\section{References}

[1] Yang JD, Roberts LR. Hepatocellular carcinoma: A global view.Nat Rev Gastroenterol Hepatol,2010,7(8):448-58

[2] Jemal A, Bray F, Center MM, et al. Global cancer statistics.CA Cancer J Clin,2011,61(2):69-90

[3] Llovet JM, Bruix J. Molecular targeted therapies in hepatocellular carcinoma. Hepatology,2008,48(4):1312-27

[4] Abou-Alfa GK, Johnson P, Knox JJ, et al. Doxorubicin plus sorafenib vs doxorubicin alone in patients with advanced hepatocellular carcinoma: a randomized trial. JAMA,2010,304(19):2154-60

[5] Yauch RL, Hemler ME. Specific interactions among transmembrane 4 superfamily (TM4SF) proteins and phosphoinositide 4-kinase. Biochem J,2000,351(pt 3):629-37

[6] Serru V, Dessen P, Boucheix C, et al. Sequence and expression of seven new tetraspans. Biochim Biophys Acta,2000,1478(1):159-63

[7] Zhu J, Zhou K, Hao JJ, et al. Regulation of cortactin/ dynamin interaction by actin polymerization during the fission of clathrin-coated pits. J Cell Sci,2005,118(Pt 4):807-17

[8] Kirkbride KC, Sung BH, Sinha S, et al. Cortactin: a multifunctional regulator of cellular invasiveness. Cell Adh Migr,2011,5(2):187-98

[9] Chen L, Wang ZW, Zhu JW, et al. Roles of cortactin, an actin polymerization mediator, in cell endocytosis. Acta Biochim Biophys Sin (Shanghai),2006,38(2):95-103

[10] Higgs HN, Pollard TD. Regulation of actin filament network formation through Arp2/3 complex: activation by a diverse array of proteins. Annu Rev Biochem,2001,70:649-76

[11] Krueger EW, Orth JD, Cao H, et al. A dynamin-cortactinArp2/3 complex mediates actin reorganization in growth factor-stimulated cells. Mol Biol Cell,2003,14(3):108596

[12] Chuma M, Sakamoto M, Yasuda J, et al. Overexpression of cortactin is involved in motility and metastasis of hepatocellular carcinoma. J Hepatol,2004,41(4):629-36

[13] Huang X, Ji J, Xue H, et al. Fascin and cortactin expression is correlated with a poor prognosis in hepatocellular carcinoma. Eur J Gastroenterol Hepatol,2012,24(6):633-9

[14] Chen L, Li X, Wang GL, et al. Clinicopathological significance of overexpression of TSPAN1, Ki67 and CD34 in gastric carcinoma. Tumori,2008,94(4):531-8

[15] Bennett G, Sadlier D, Doran PP, et al. A functional and transcriptomic analysis of NET1 bioactivity in gastric cancer. BMC Cancer, 2011, 11:50.

[16] Chen L, Wang Z, Zhan X, et al. Association of TSPAN1 gene expression with human hepatocellular carcinoma. Int J Surg Pathol, 2007, 15: 346-53.

[17] Chen L, Yuan D, Wang GL, et al. Clinicopathological significance of expression of Tspan-1, Jabl and 
p27 in human hepatocellular carcinoma. J Korean Med Sci,2010,25(10):1438-42

[18] Shen SQ, Li K, Zhu N, et al. Expression and clinical significance of NET-1 and PCNA in hepatocellular carcinoma. Med Oncol,2008,25(3):341-5

[19] Chen L, Zhu YY, Zhang XJ, et al. TSPAN1 protein expression: A significant prognostic indicator for patients with colorectal adenocarcinoma. World J Gastroenterol, 2009,15:2270-6

[20] Chen L, Yuan D, Zhao R, et al. Suppression of TSPAN1 by RNA interference inhibits proliferation and invasion of colon cancer cells in vitro. Tumori,2011,96(5):744-50

[21] Zhang J, Wang J, Chen L, et al. Expression and function of NET-1 in human skin squamous cell carcinoma. Arch Dermatol Res,2014,306(4):385-97

[22] Chen L, Zhu Y, Li H, et al. Knockdown of TSPAN1 by RNA silencing and antisense technique inhibits proliferation and infiltration of human skin squamous carcinoma cells. Tumori,2010,96(2):289-95

[23] Wollscheid V, Kuhne-Heid R, Stein I, et al. Identification of a new proliferation associated protein NET-1/ C418 characteristic for a subset of high graed cervical intraepithelial neoplasia and cervical carcinomas. Int J Cancer, 2002, 99:771-5.

[24] Bergé M, Bonnin P, Sulpice E, et al. Small interfering RNAs induce target-independent inhibition of tumor growth and vasculature remodeling in amouse model of hepatocellular carcinoma. Am J Pathol, 2010,177:3192201.

[25] Li X, Pan Y, Fan R, et al. Adenovirus-delivered CIAPIN1 small interfering RNA inhibits HCC growth in vitro and in vivo. Carcinogenesis,2008,29(8):1587-93

[26] World Health Organization classification of tumors. In: Stanley RH, Lauri AA, Editors. Pathology and Genetics Tumors of the Digestive System, Lyon: IARC Press, 2004:104.

[27] Wang GL, Chen L, Wei YZ, et al. The effect of NET1 on the proliferation, migration and endocytosis of the SMMC-7721 HCC cell line. Oncol Rep,2012,27(6):194452

[28] Kowalski JR, Egile C, Gil S, et al. Cortactin regulates cell migration through activation of N-WASP. J Cell
Sci,2005,118(Pt 1):79-87

[29] Polo JM, Dell'oso T, Ranuncolo SM, et al. Specific peptide interference reveals BCL6 transcriptional and oncogenic mechanisms in B-cell lymphoma cells. Nat Med,2004,10(12):1329-35

[30] Menendez JA, Vellon L, Mehmi I, et al. Inhibition of fatty acid synthase (FAS) suppresses HER2/neu (erbB-2) oncogene overexpression in cancer cells. Proc Natl Acad Sci U S A,2004,101(29):10715-20

[31] Watanabe T, Sudoh M, Miyagishi M, et al. Intracellulardiced dsRNA has enhanced efficacy for silencing HCV RNA and overcomes variation in the viral genotype. Gene Ther,2006,13(11):883-92

[32] Sano M, Li H, Nakanishi M, et al. Expression of long anti-HIV-1 hairpin RNAs for the Generation of multiple siRNAs: advantages and limitations. Mol Ther,2008,16(1):170-7

[33] Hossbach M, Gruber J, Osborn M, et al. Gene silencing with siRNA duplexes composed of target-mRNA-complementary and partially palindromic or partially complementary single-stranded siRNAs. RNA Biol,2006,3(2):829

[34] Tiemann K, Höhn B, Ehsani A, et al. Dual-targeting siRNAs. RNA,2010,16(6):1275-84

[35] Yuan BZ, Zhou X, Zimonjic DB, et al. Amplification and overexpression of the EMS 1 oncogene, a possible prognostic marker, in human hepatocellular carcinoma. $J \mathrm{Mol}$ Diagn,2003,5(1):48-53

[36] Kasibhatla S, Tseng B. Why target apoptosis in cancer treatment? Mol Cancer Ther, 2003, 2: 573-80.

[37] Varfolomeev E, Vucic D. Inhibitor of apoptosis proteins: fascinating biology leads to attractive tumor therapeutic targets. Future Oncol,2011,7(5):633-48

[38] Qiu DM, Wang GL, Chen L, et al. The expression of beclin-1, an autophagic gene, in hepatocellular carcinoma associated with clinical pathological and prognostic significance. BMC Cancer,2014,14(1):327

[39] Chang CI, Kang HS, Ban C, et al. Dual-target gene silencing by using long, synthetic siRNA duplexes without triggering antiviral responses. Mol Cells,2009,27(6):689-95

Received 3 May 2017, Revised 21 May 2017, Accepted 05 June 2017 